\title{
Value of Emergency Nursing Procedures in Improving the First Aid Efficiency and Outcomes of Comatose Patients with Cerebral Hemorrhage
}

\author{
Yanli Jiao* \\ Suzhou Hospital of Traditional Chinese Medicine Affiliated to Nanjing University of Chinese Medicine, Suzhou 215009, \\ Jiangsu Province, China \\ *Corresponding author: Yanli Jiao, 86309947@qq.com \\ Copyright: ( $) 2022$ Author(s). This is an open-access article distributed under the terms of the Creative Commons Attribution License (CC \\ BY 4.0), permitting distribution and reproduction in any medium, provided the original work is cited.
}

\begin{abstract}
Objective: To determine the application value of emergency nursing in improving the efficiency and outcomes of first aid for comatose patients with cerebral hemorrhage. Methods: Forty comatose patients with cerebral hemorrhage treated in Suzhou Hospital of Traditional Chinese Medicine from August 2018 to June 2019 were selected as the research subjects in this study; the patients were divided into two groups, a control group and a study group, by lot; the patients in the control group received nursing by conventional means, while emergency nursing procedures were provided to the patients in the study group; the efficiency of first aid and the outcomes of the two groups were compared; the application value of emergency nursing procedures was analyzed. Results: The first aid efficiency of the study group was significantly higher than that of the control group; the condition evaluation, reception time, triage time, and rescue time of the study group were significantly lower than those of the control group; the treatment outcomes of the study group were significantly better than those of the control group $(P<0.05)$. Conclusion: Based on the results, compared with conventional nursing methods, the implementation of emergency nursing procedures for comatose patients with cerebral hemorrhage helps to improve the efficiency of first aid and the outcomes of the treatment; it has high clinical application value and is worthy of popularization as well as application in clinical practice.
\end{abstract}

Keywords: Emergency nursing procedures; Cerebral hemorrhage with coma; Efficiency of first aid; Outcomes of first aid

Online publication: January 19, 2022

\section{Introduction}

Cerebral hemorrhage is a cerebrovascular disease caused by cerebral ischemia which results in nerve dysfunction. It is relatively common in clinical practice and has a high incidence, mainly occurring among the elderly. With the rapid development of society, the accelerated pace of the city, and the increasing stress, the incidence among middle-aged groups has shown a substantial rise in trend ${ }^{[1]}$. In cerebral hemorrhage, the thrombus in the cerebral nerve tissue may fall off and travel through the systemic circulation to cerebral vessels, resulting in the blockage of main cerebral vessels and inducing acute cerebral infarction ${ }^{[2]}$. Many clinical studies have proven that in cerebral hemorrhage, the blood glucose levels are significantly raised, the anaerobic glycolysis of glycogen is promoted, there is continuous accumulation of acidic metabolites, the activity of protease is inhibited, and many peroxy free radicals are generated, causing damage to the neurons in the brain and inducing various complications ${ }^{[3]}$. When the blood vessels in the cranial cavity are blocked, it will lead to the failure of cerebral blood circulation, interrupt the blood and oxygen supply 
to cerebral nerves, cause hypoxia and swelling of cerebral neurons, compress the small blood vessels around the nerve, as well as increase the probability of cerebral hemorrhage transformation, which will not only affect limb coordination and motor function, but also threaten the patient's life in serious cases, causing damage to the patient's physical and mental health ${ }^{[4]}$. Patients with cerebral hemorrhage have a sudden onset, and their condition changes in a short time. Secondary cerebral hemorrhage may occur. A large amount of bleeding may endanger the patient's life. Coma is one of the common complications of patients with intracerebral hemorrhage. If a patient with cerebral hemorrhage does not wake up in time, the patient may be in a vegetative state. The routine first aid process does not only have a slow diagnosis time, but also a low inspection efficiency, thus missing the best rescue opportunity for comatose patients with cerebral hemorrhage. After treatment, most patients would still have a series of sequelae, which cannot meet the growing nursing needs of patients ${ }^{[5]}$. Therefore, timely diagnosis and treatment can help reduce the probability of cerebral infarction caused by cerebral hemorrhage. According to clinical research, timely and efficient implementation of emergency nursing procedures for comatose patients with cerebral hemorrhage can shorten the diagnosis, transportation time, and rescue time, change the treatment outcomes, reduce the probability of disability and death, effectively improve the rescue efficiency, establish a good foundation for follow-up treatment, and promote the patient's brain and nervous system to return to normal along with their prognosis.

Emergency nursing is a comprehensive, professional, and scientific new nursing model, which makes the nursing process standardized, efficient, and scientific. It can improve the efficiency of first aid as well as the level and quality of nursing, provide basic guarantee for follow-up treatment, and satisfy the patients. In this study, 40 comatose patients with intracerebral hemorrhage were taken as the research subjects, sand the clinical effects of emergency nursing procedures were analyzed and sorted out.

\section{Materials and methods}

\subsection{General information}

Forty comatose patients with cerebral hemorrhage, treated in Suzhou Hospital of Traditional Chinese Medicine from August 2018 to June 2019, were selected as the research subjects. The patients were randomly divided into two groups, the control group and the study group, with 20 patients in each group. Conventional nursing methods were used for the patients in the control group. There were 11 male patients and 9 female patients, age ranging from 43 to 76 years old, with an average age of $59.52 \pm 7.37$ years old, and the time from onset to admission was $2.12 \pm 0.14$ hours. In the study group, there were 12 male patients and 8 female patients, age ranging from 45 to 78 years old, with an average age of $60.76 \pm 8.12$ years old, and the time from onset to admission was $2.31 \pm 0.16$ hours. There was no significant difference between the two groups $(P>0.05)$.

\subsection{Inclusion and exclusion criteria}

The inclusion criteria were as follows: (1) patients who were clinically diagnosed with cerebral hemorrhage coma; (2) the time from onset to admission was < 24 hours; (3) patients and their family members volunteered to participate in this study.

The exclusion criteria were as follows: (1) patients with severe heart, liver, lung, or kidney diseases;

(2) patients whose vital signs had no rescue significance at admission; (3) patients and their families who disagreed with the implementation of emergency nursing procedures.

\subsection{Methods}

The control group received routine nursing. Upon diagnosis, the condition of each patient was preliminarily 
evaluated, ECG monitoring was performed, oxygen was given according to situation, the patient's vital signs were monitored, and the rescue process was assisted.

Emergency nursing was carried out for the patients in the study group. Several measures were taken based on the steps below.

(1) Establish an emergency nursing procedure team, improve various emergency nursing procedures, select experienced emergency medical staffs, strengthen the training of nursing knowledge prior to actual work, improve the first-aid professional skills and adaptability of medical staffs, place medical devices and drugs at fixed points, and keep them with special personnel, clarify the division of responsibilities among team members, and carry out emergency nursing procedures in an orderly manner.

(2) Upon receiving an emergency call, do a quick visit within 5 minutes. After arriving at the site, provide first aid; during transportation, keep the patient lying on his or her back, supply conventional oxygen therapy, and always pay attention to the changes of vital signs. Then, contact the hospital triage desk to inform the patient's condition, so as to guide their preparations based on the patient's condition (e.g., antihypertensive drugs, thrombolytic drugs, and other relevant rescue items).

(3) Upon admission, the nursing staffs at the pre-examination desk shall assess the severity of the patient's condition, open a green channel for the patient, transfer the patient to the emergency room within three minutes, and hand over the patient to the emergency personnel in the hospital.

(4) After being transferred to the emergency room, the emergency nursing staffs shall assist the attending doctor to establish venous channels, collect blood samples, conduct brain computed tomography (CT) examination, evaluate the condition of the patient, and then initiate thrombolytic therapy. Thrombolytic therapy is a common treatment for intracerebral hemorrhage, as it can effectively open up the blocked blood vessels and restore the normal blood and oxygen supply to brain tissues. However, this therapy has a certain risk of cerebral infarction. After anoxic death, the neurons in the infarct focus promotes the generation of a large number of oxygen free radicals, resulting in oxidative stress injury to local tissues. The secondary injury after thrombolytic therapy will aggravate the compression on neurons and increase the risk of rebleeding. Therefore, it is necessary to use thrombolytic therapy carefully especially for comatose patients with cerebral hemorrhage, grasp the applicability of thrombolytic drugs for patients, and reduce the risk of cerebral infarction.

(5) Ask the family members whether the patient has severe drug allergy, hypertension, diabetes, or other comorbidities, and provide psychological counseling to help relieve their anxiety and promote their cooperation with emergency nursing procedures.

(6) During thrombolytic therapy, pay close attention to the changes of the patient's vital signs, such as heart rate, blood pressure, and body temperature, observe whether the patient's pupils and pulse are abnormal, as well as prevent secondary damage to the brain as a result of cerebral ischemia.

\subsection{Observation indicators}

The emergency outcomes and efficiency of the two groups were compared.

\subsection{Statistical analysis}

The data in this study were calculated and analyzed by SPSS 23.0. P $<0.05$ indicates that there is statistical 
significance. The measurement data were tested by t-test and expressed in mean \pm standard deviation $(\overline{\mathrm{x}} \pm$ s), while the counting data were expressed in n (\%), with $X^{2}$ as the main parameter.

\section{Results}

\subsection{Outcomes of first aid}

The outcomes of first aid of the two groups are shown in Table 1.

Table 1. Outcomes of the two groups [n (\%)]

\begin{tabular}{cccc}
\hline Group & Rescue success rate & Disability rate & Mortality rate \\
\hline Study group $(\mathrm{n}=20)$ & $19(95.00 \%)$ & $1(5.00 \%)$ & $1(5.00 \%)$ \\
Control group $(\mathrm{n}=20)$ & $14(70.00 \%)$ & $7(35.00 \%)$ & $6(30.00 \%)$ \\
$X^{2}$ value & 4.329 & 5.625 & 4.329 \\
$P$ value & 0.037 & 0.018 & 0.037 \\
\hline
\end{tabular}

\subsection{Efficiency of first aid}

The comparison of the efficiency of first aid between the two groups is shown in Table 2.

Table 2. Comparison of the efficiency of first aid between the two groups $(\overline{\mathrm{x}} \pm \mathrm{s}, \mathrm{min})$

\begin{tabular}{ccccc}
\hline Group & Evaluation of condition & Reception time & Triage time & Rescue time \\
\hline Study group $(\mathrm{n}=20)$ & $0.81 \pm 0.23$ & $4.21 \pm 0.83$ & $3.15 \pm 0.37$ & $35.46 \pm 2.62$ \\
Control group $(\mathrm{n}=20)$ & $1.24 \pm 0.68$ & $5.34 \pm 1.32$ & $4.72 \pm 1.34$ & $40.17 \pm 3.15$ \\
$t$ value & 2.679 & 3.241 & 5.051 & 5.141 \\
$P$ value & 0.011 & 0.002 & 0.000 & 0.000 \\
\hline
\end{tabular}

\section{Discussion}

Cerebral hemorrhage is a common brain disease in neurosurgery. The pathological basis of this disease is a disorder in cerebral blood supply and internal circulation, resulting in nerve damage. If the patient is not treated in time, it may endanger the patient's life. The development and change in the condition of a comatose patient with cerebral hemorrhage is complex, and the rescue is difficult, so there is not much time for the emergency nursing staffs. The conventional nursing method has low rescue efficiency and high disability rate as well as mortality rate, which is not conducive to grasping the golden opportunity of rescue, thus resulting in unsatisfactory treatment effect. Clinical research shows that the implementation of scientific and effective first-aid nursing procedures can reduce the damage to patients' neuronal tissues, improve the first aid effect, shorten the rescue time, reduce the disability rate and mortality rate, as well as change the treatment outcomes.

Emergency nursing is a professional first aid mode. The team members have clear responsibilities, carry out their responsibilities in an orderly manner, and assume targeted treatment measures according to the changes in the patient's condition, thus shortening the rescue time, improving the first aid efficiency, changing the treatment outcomes, and promoting the smooth development of first aid. In this study, 40 comatose patients with cerebral hemorrhage were selected as the research subjects, in which 20 patients received routine nursing and the remaining received emergency nursing. The first aid efficiency and outcomes of the two groups were analyzed and compared. The research results showed that the first aid efficiency of the study group was significantly higher than that of the control group, in which the condition 
evaluation, reception time, triage time, and rescue time of the study group were lower than those of the control group; the emergency outcomes of the study group were also significantly better than those of the control group, indicating that emergency nursing procedures can significantly improve the first aid efficiency, clarify the understanding of patients and their family members about cerebral hemorrhage coma, and improve the overall satisfaction of patients with the nursing work. Emergency nursing is a scientific and effective nursing method to improve nursing efficiency and patient rescue efficiency. It is suitable for the rescue and nursing of comatose patients with cerebral hemorrhage. It strives for the best treatment opportunity for patients, improves the treatment outcomes, provides a good foundation for follow-up treatment, and has high clinical value. Therefore, it is worthy of further promotion in clinical practice.

In conclusion, the application of emergency nursing procedures to the nursing of comatose patients with cerebral hemorrhage helps to improve the first aid efficiency and the treatment outcomes. Good clinical results have been observed through emergency nursing; thus, it is worthy of popularization and application in clinical practice.

\section{Disclosure statement}

The author declares that there is no conflict of interest.

\section{References}

[1] Guo L, 2018, Analysis of the Function of Emergency Nursing Procedure on Improving the First aid Efficiency and Improving the First Aid Outcome of Comatose Patients with Cerebral Hemorrhage. China Health Standard Management, 9(8): 153-155.

[2] $\mathrm{Hu}$ Y, 2019, Analysis of the Effect of Emergency Nursing Procedure on Improving the First aid Efficiency and Improving the First Aid Outcome of Comatose Patients with Cerebral Hemorrhage. Special Health, 2019(14): 180-181.

[3] Zhang Z, 2019, Clinical Effect of Emergency Nursing Procedure on Improving the First aid Efficiency and Improving the First Aid Outcome of Comatose Patients with Cerebral Hemorrhage. China Health Care \& Nutrition, 29(27): 306.

[4] Li H, Wan L, 2019, Application Effect of Emergency Nursing Management Procedure in Patients with Coma After Cerebral Hemorrhage. China Modern Medicine, 2019(19): 219-221.

[5] Gao X, 2019, Application Evaluation of Optimizing Emergency Nursing Process in the Rescue of Comatose Patients with Cerebral Hemorrhage. Women's Health Research. 2019(21): 179-180.

\section{Publisher's note}

Bio-Byword Scientific Publishing remains neutral with regard to jurisdictional claims in published maps and institutional affiliations. 